\title{
A Prototype of Virtual Traffic Lights on Android-based Smartphones
}

\author{
Manuel Nakamurakare ${ }^{\star}$, Wantanee Viriyasitavat ${ }^{\dagger \ddagger}$, and Ozan K. Tonguz ${ }^{\dagger}$ \\ *Information Networking Institute, Carnegie Mellon University, Pittsburgh, PA 15213-3890, USA \\ ${ }^{\dagger}$ Department of Electrical and Computer Engineering, Carnegie Mellon University, Pittsburgh, PA 15213-3890, USA \\ ${ }^{\ddagger}$ Faculty of Information and Communication Technology, Mahidol University, Thailand 10400 \\ E-mail: mnakamur@andrew.cmu.edu, wviriyas@ece.cmu.edu, tonguz@ece.cmu.edu
}

\begin{abstract}
Virtual Traffic Lights (VTL) is a recently proposed self-organizing traffic control scheme that has the potential to mitigate traffic congestion in urban areas. This paper reports a prototype design effort on Virtual Traffic Lights using Androidbased smartphones. The experiments performed show the feasibility of implementing VTL using smartphones' WiFi devices.
\end{abstract}

Keywords-Vehicle-to-vehicle communications, ad hoc networks, self-organizing networks, smartphone applications

\section{INTRODUCTION}

A promising approach for mitigating traffic congestion was proposed recently [1]. This new approach is known as Virtual Traffic Lights (VTL) and it leverages the envisioned ubiquitous presence of vehicle-to-vehicle (V2V) communications. While the technology for V2V communications, namely Dedicated Short Range Communications (DSRC) technology, has been standardized and is expected to be a mandate in the next 23 years in the United States, the current availability and the global use of this technology is very limited.

Because of the similarity between DSRC standard and the IEEE 802.11a and the proliferation of Wi-Fi hand-held devices, in this paper, we propose to implement the VTL concept as a smartphone application which makes use of the already available hardware components such as navigation system (i.e. GPS and map), radio device (i.e., WiFi), a processing unit (i.e., microcontroller), and a display. Note that while the VTL design and architecture can be applied to all smartphone development platforms, in this work, we focus on implementing it on Android-based smartphones due to the following reasons: i) cost: all iOS developers are required to go through the iOS Developer Program which costs at least $\$ 99$ to enroll [2]; ii) dominant share of market: as of the end of 2012, Android had a $68.3 \%$ market share [3]; iii) platform flexibility: the development can be done on any operating system; and iv) availability of tools and online support system.

It is our hope that the Android-based VTL implementation will play an important role in demonstrating the benefits of the VTL paradigm and encourage researchers as well as policy makers to take a serious look at this new way of dealing with urban traffic congestion.

\section{PRinciple of Operation}

The premise of the Android-based implementation presented in this paper is the self-organized traffic control

This research was supported in part by the T-SET University Transportation Center sponsored by the US Department of Transportation under Grant No. DTRT12-G-UTC11.

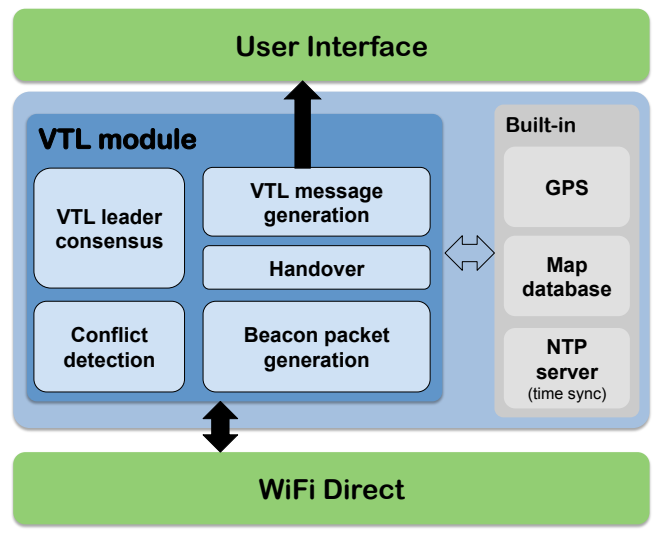

Figure 1. Design and architecture of Virtual Traffic Light implementation on Android-based smartphones.

paradigm proposed in [1]. In this paradigm, conflicts at intersections are detected and resolved in an ad hoc manner without any help from infrastructure. By using vehicle-tovehicle communications (i.e., each vehicle sends out periodic hello messages to inform nearby vehicles of its presence, current position, and velocity), each vehicle can determine if there is an ensuing conflict at the intersection it is about to approach. When a conflict is detected, vehicles involved in the conflict perform the following three steps:

\section{A. Leader Election Process}

As vehicles approach the same intersection (when, a conflict is detected), vehicles on the same road must agree on electing one of them (usually the closest one to the intersection) to act as the cluster leader and all cluster leaders must agree on electing one of the cluster leaders to act as the VTL leader for the intersection. Once the VTL leader has been identified, it announces its leadership to all cluster leaders who, upon receiving such announcement message, reply with acknowledgment packets. The elected leader will serve as temporary traffic light infrastructure and is responsible for creating and broadcasting traffic light information. Other vehicles act as passive nodes, listen to and obey the traffic light information broadcasted from the leader. To avoid unnecessary leader election process, the leader is presented with red light and stops at the intersection while leading it.

\section{B. Generation of Traffic Light Information}

Once a leader is elected, it determines how long each approaching direction should receive the right of way (i.e., 
phase layout of the traffic light). This phase layout could be pre-programmed or dynamically configured based on several parameters such as the amount of traffic in each direction, level of congestion at the intersection, priority of roads, etc. To enable a fair utilization of the intersection, the number of cars waiting in each road should also be taken into account. Phase preemption could also be enabled - once the VTL leader detects that the road with the green light has no additional vehicles attempting to cross the intersection, the current phase is interrupted and the green light is given to the next connecting road [1].

\section{Leader Handover}

When the green light is in the leader's lane, a new leader must be elected to maintain the virtual traffic light infrastructure. The new leader can be elected by two possible mechanisms: - i) the current leader hands over the leading task to one of the vehicles stopped before a red light at the intersection or ii) the new leader election is performed if there are no stopped vehicles at red lights.

It has been shown by extensive simulations that the aforementioned traffic control scheme (i.e., Virtual Traffic Light (VTL) system) could provide up to $60 \%$ improvement in traffic flow [1]. It should be noted that the VTL system operates under the assumption that all vehicles are equipped with DSRC radios and vehicle-to-vehicle communications problems (such as packet collisions, packet drops due to obstruction in RF propagation) are not severe and do not disrupt the VTL leader election. In addition, all vehicles are assumed to be equipped with a GPS device that shares the same digital map and has lane-level accuracy.

\section{Virtual Traffic Light ARChitecture AND PROTOTYPE DESIGN}

Overview of the design and architecture used to implement the VTL protocol is depicted in Figure 1. In addition to the five key submodules implemented within the VTL module, the implementation includes the user interface component, makes use of and operates based on the input provided by the built-in modules such as GPS, Map database, and NTP server (for time synchronization purpose ${ }^{1}$ ). Two different map formats are used in the implementation: XML format [4] used in VTL module and GoogleMap format used for display purpose. Note that, in this work, the WiFi Direc ${ }^{2}$ (i.e., the WiFi infrastructureless mode in Android) is used as the underlying means of communications.

Figure 2 depicts the state machine design of our implementation. The VTL module can be in one of the four states as shown in the figure. Upon approaching an intersection, the vehicle, based on its own location and locations of its neighbors obtained from beacon packets (see Figure 3(i)),

\footnotetext{
${ }^{1}$ Note that we have implemented three different time synchronization methods (i.e., through GPS clock, wireless provider clock, and NTP server). Although the NTP server can best address the time synchronization issue, the experiments have shown that the time difference between two phones can be as large as $900 \mathrm{~ms}$. The time synchronization problem thus requires further investigation.

${ }^{2}$ Only Android 4.0 and later versions can support the WiFi Direct mode.
}

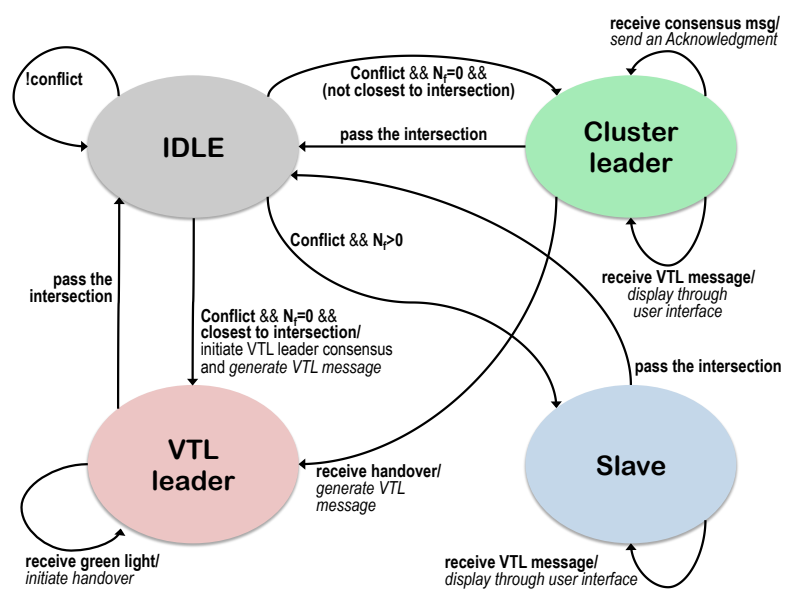

Figure 2. State machine of the VTL implementation in Android-based smartphones. $N_{f}$ denotes the number of vehicles in the front that are approaching the same intersection.

determines if there is an ensuing conflict at the intersection it is about to approach. In the case where a conflict is detected, the vehicle determines, again based on its location and location of its neighbors, whether or not it should serve as the Cluster Leader for the road it travels. In other words, if it is the closest vehicle to the intersection in its own cluster, the vehicle will be elected as the cluster leader; otherwise, it will act as a passive node and obey the traffic light information (see Figure 3(iv)) broadcasted from the VTL leader or the cluster leader. It is worth pointing out that the cluster leader may become the VTL leader in one of the two situations: i) among the cluster leaders on the other approaching streets, the vehicle is the closest vehicle to the intersection; or ii) the vehicle receives the handover message from the current VTL leader. In the scenario where neither of these two conditions are met, the vehicle remains as the cluster leader; it sends an acknowledgment packet (see Figure 3(iii)) upon receiving VTL Leader packet (see Figure 3(ii)) from the elected VTL leader and obeys the traffic light information broadcasted from the VTL leader.

The VTL leader, on the other hand, is responsible for computing traffic signal plan for the intersection it serves, and broadcasting the traffic light information to other vehicles. Upon receiving a green light and when it is ready to cross the intersection, the VTL leader initiates a handover process to delegate the VTL leader responsibility to one of the other cluster leaders at the intersection.

\section{DEMONSTRATION}

The main objective of the demonstration is to show the operation of the VTL module implemented on Android smartphones. Three phones - two Nexus 4 running Android 4.2 and Galaxy S 3 mini running Android 4.1 - are used in the demonstration and their screenshots are shown in Figure 4. In order to verify the operation of the implemented module, we isolate the communications and GPS problems by performing the experiment in a simple and ideal indoor scenario in which the GPS locations of each vehicle (i.e., phone) are emulated and three phones are put next to one another as shown in Figure 4. 


Beacon Packet
i) \begin{tabular}{|c|c|c|c|c|c|c|c|}
$\begin{array}{c}\text { Pkt } \\
\text { Type }\end{array}$ & $\begin{array}{c}\text { Unique } \\
\text { Pkt ID }\end{array}$ & Timestamp & IP Address & Coordinates & Dir. & $\begin{array}{c}\text { Lane } \\
\text { ID }\end{array}$ & $\begin{array}{c}\text { VTL } \\
\text { Leader? }\end{array}$ \\
\hline \\
VTL message Packet \\
ii) \begin{tabular}{c|c|c|c|c|} 
Pkt & Unique \\
Type & Pkt ID
\end{tabular} & Timestamp & IP Address & Signaling plan \\
\hline
\end{tabular}

\begin{tabular}{|c|c|c|c|c|c|}
\hline \multirow[b]{2}{*}{ iii) } & \multicolumn{4}{|c|}{ VTL Leader Packet } & \\
\hline & $\begin{array}{c}\text { Pkt } \\
\text { Type }\end{array}$ & $\begin{array}{l}\text { Unique } \\
\text { Pkt ID }\end{array}$ & Timestamp & IP Address & \\
\hline \multicolumn{6}{|c|}{ Leader Acknowledgment Packet } \\
\hline iv) & $\begin{array}{l}\text { Pkt } \\
\text { Type }\end{array}$ & $\begin{array}{l}\text { Unique } \\
\text { Pkt ID }\end{array}$ & Timestamp & IPAddress & $\begin{array}{c}\text { Le of } \\
\text { Leader } \\
\text { pkt }\end{array}$ \\
\hline
\end{tabular}

Figure 3. Four different types of packet implemented in the VTL protocol.

As depicted in the figure, the user interface consists of 5 elements: i) map, ii) vehicle icons that represent current locations of itself (i.e., indicated by black color) and its neighbors, iii) traffic light color displayed to the vehicle, iv) control buttons to manually change vehicle locations, and v) debugging information to show the status of the VTL operation.
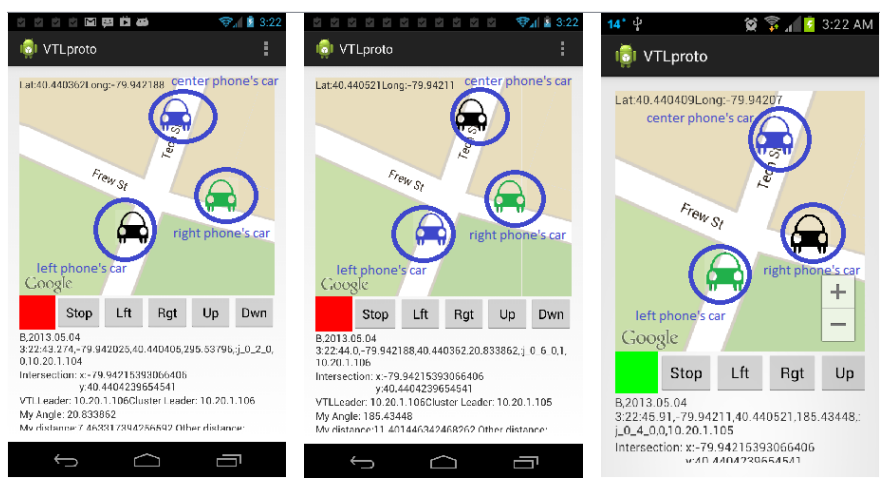

Figure 4. VTL user interface on Android-based smartphones. In this scenario, a total of three phones are used. The VTL module in the phone is able to locate itself (indicated by the vehicle in black color) and nearby vehicles (indicated by green and blue colors).

Screenshots shown in Figure 4 depict the scenario where three vehicles are approaching the intersection from three different directions (i.e., the left, middle, and right vehicles/phones travel in northbound, southbound, and westbound directions, respectively). Since there is one vehicle in each direction, all three vehicles serve as the cluster leader except the left vehicle who also serves as the VTL leader because it is the closest vehicle to the intersection ${ }^{3}$. The elected VTL leader then presents a red light to its approach and a green light to the orthogonal direction. As a result, the left and middle vehicles are presented with a red light where the right vehicle who travels on the orthogonal road is presented with a green light.

In addition to demonstrating the VTL operation, a set of measurements have been carried out to measure the packet latency which is defined as the duration of time from which a beacon packet is sent from the VTL application in one phone to the time the same packet is received by the VTL

\footnotetext{
${ }^{3}$ The VTL leader election process which consists of broadcasting a VTL leader packet by the VTL leader and Leader Acknowledgment packets by other cluster leaders is performed in the background.
}

application in the other phone. Note that since the receiver thread is not always active (i.e., it becomes active every $n \mathrm{~ms}$ where $n$ is the receiver sleep time), the beacon packet latency significantly varies with the value of $n$. Observe from Table I that even with the large receiver sleep time of 1 second, the packet latency is small and remains below 0.1 second. This, in turn, suggests that it may be feasible to implement the VTL operation on smartphones; i.e., by the time a vehicle receives a beacon packet, the transmitting vehicle moves less than 1 meter. In addition to latency measurement, it is necessary to measure the communication range provided by the Android's WiFi radio for determining the feasibility of the Android-based VTL implementation.

\begin{tabular}{|c|c|c|}
\hline Receiver sleeptime (ms) & Average (ms) & Std. dev. $(\mathrm{ms})$ \\
\hline 25 & 2.54 & 1.63 \\
\hline 100 & 9.97 & 6.31 \\
\hline 1000 & 77.05 & 73.87 \\
\hline
\end{tabular}

Table I

PACKET LATENCY FOR DIFFERENT RECEIVER SLEEPTIME

\section{CONCLUSion ANd Future Work}

In this paper, we present the Virtual Traffic Lights (VTL) implementation on smartphones using Android OS. The VTL concept is a self-organizing traffic control concept which aims to alleviate the urban traffic congestion problem by leveraging the vehicle-to-vehicle communications. By communicating among themselves, vehicles, in an ad hoc manner, are able to resolve conflicts at intersections and establish the "right of way". Previous simulation results have shown that the VTL can provide up to $60 \%$ improvement in traffic flow. In order to demonstrate benefits of the VTL in a real-world scenario, in this paper, we propose to implement the VTL on smartphones using Android OS, mainly due to the proliferation of smartphones and the dominant market share of Android operating system.

The promising experimental results reported in this paper show that the VTL concept can be implemented using hardware available in the current smartphones. While there are other issues (such as fault-tolerance and security issues) that need further investigation, we believe that the VTL prototype presented in this paper clearly demonstrates the feasibility of implementing VTL concept. It is our hope that the promising results presented in this paper will provide further motivation for policy makers to pass legislation for the use of VTL as a compelling solution for mitigating urban traffic congestion.

\section{REFERENCES}

[1] M. Ferreira, R. Fernandes, H. Conceição, W. Viriyasitavat, and O. K. Tonguz, "Self-organized traffic control," in the ACM international workshop on VehiculAr InterNETworking (VANET), 2010, pp. 85-90.

[2] iOS Developer Program, "https://developer.apple.com/programs/ios/," May 2013.

[3] International Data Corporation Press Release, "Worldwide Mobile Phone Growth Expected to Drop to 1.4\% in 2012 Despite Continued Growth Of Smartphones," http://www.idc.com/getdoc.jsp?containerId=prUS23818 212\#.UL4uOpPjmMR, December 2012.

[4] "SUMO Road Networks," http://sumo.sourceforge.net/doc/current/docs/ userdoc/Networks/SUMO_Road_Networks.html, 2013. 